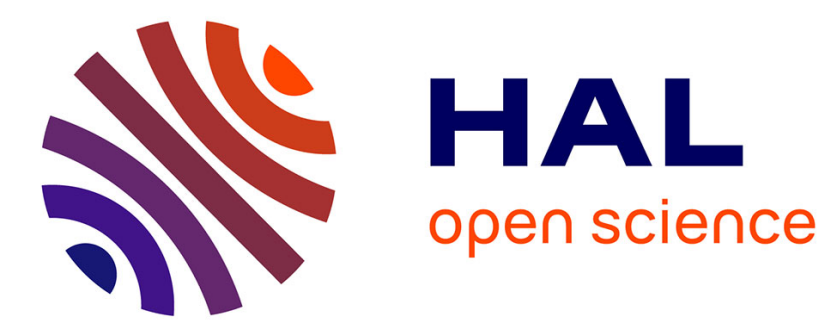

\title{
Novel reactions catalysed by antibodies
}

Beatrice Golinelli-Pimpaneau

\section{To cite this version:}

Beatrice Golinelli-Pimpaneau. Novel reactions catalysed by antibodies. Current Opinion in Structural

Biology, 2000. hal-03281465

\section{HAL Id: hal-03281465 \\ https://hal.science/hal-03281465}

Submitted on 8 Jul 2021

HAL is a multi-disciplinary open access archive for the deposit and dissemination of scientific research documents, whether they are published or not. The documents may come from teaching and research institutions in France or abroad, or from public or private research centers.
L'archive ouverte pluridisciplinaire HAL, est destinée au dépôt et à la diffusion de documents scientifiques de niveau recherche, publiés ou non, émanant des établissements d'enseignement et de recherche français ou étrangers, des laboratoires publics ou privés. 


\section{Commentary Novel reactions catalysed by antibodies Béatrice Golinelli-Pimpaneau}

\begin{abstract}
New structural data on nonhydrolytic antibody catalysts gained over the past two years confirm that antibodies elicited against transition state analogues function by differential stabilisation of the transition-state over the ground state through electrostatic, van der Waals, cation $-\pi$ and hydrogen-bonding interactions. The lack of chemical catalysis correlates with the low catalytic efficiency. Novel strategies that precisely position a key functional residue in the antibody catalyst combining site have therefore emerged, as demonstrated by crystallographic studies. Whereas antibodies with a bulky residue at position $\mathrm{H} 100 \mathrm{c}$ of hypervariable loop $\mathrm{H} 3$ adopt different cavity shapes, other antibodies share a common deep combining site. This structural restriction might reflect the use of similar hydrophobic haptens to generate the antibody; novel hapten design or new immunisation strategies may, in the future, lead to more structurally diversified active sites.
\end{abstract}

\section{Addresses \\ Laboratoire d'Enzymologie et Biochimie Structurales, CNRS Bât 34, 1 avenue de la Terrasse, 91198 Gif-sur-Yvette Cedex, France; e-mail: beatrice.golinelli@lebs.cnrs-gif.fr}

Current Opinion in Structural Biology 2000, 10:697-708

0959-440X/00/\$ - see front matter

(C) 2000 Elsevier Science Ltd. All rights reserved.

$\begin{array}{ll}\text { Abbreviations } \\ \text { CDR } & \text { complementarity-determining region } \\ \text { PDB } & \text { Protein Data Bank } \\ \mathrm{V}_{\mathrm{H}} & \text { heavy chain variable domain } \\ \mathrm{V}_{\mathrm{L}} & \text { light chain variable domain }\end{array}$

\section{Introduction}

Since 1986, it has been shown that antibodies generated by immunisation with appropriate transition-state analogues are able to catalyse a variety of chemical transformations [1,2]. An examination of structural data concerning catalytic antibodies, combined with biochemical studies, elucidates their mechanisms, as well as provides insight into enhancing their catalytic efficiencies. This is necessary for these tailor-made catalysts to be used in practical applications as biochemical reagents, chemical process catalysts or therapeutics.

Previously published reviews on the structures of catalytic antibodies focused mainly on hydrolytic reactions [3-6]. Crystal structures of antibodies with novel nonhydrolytic activities have emerged these past two years (Table 1, Figure 1), including examples of antibody-catalysed disfavoured reactions, in which the binding energy serves to drive the chemical reaction in an otherwise inaccessible direction. Affinity maturation introduces somatic mutations into the antibody that increase binding affinity to the hapten. [A hapten is a small molecule that is not immunogenic by itself; however, when this molecule is conjugated to a carrier protein, it has the ability to stimulate the formation of antibodies highly specific and complementary to its structure.] The structural basis for the effect of these mutations on the catalytic rate was established in two cases by comparing the mature antibody and its germline counterpart. Structures have also validated strategies different from the classical transitionstate analogue approach that aim to generate chemically reactive residues in the combining site of antibodies.

This commentary first summarises the details about the catalytic mechanisms that have been provided by X-ray structures of antibody-hapten complexes. Then, the antibody catalytic pockets are compared through superposition of their coordinates to gain insight into the diversity of modes of binding of the hapten to the recombinant site and to evaluate the key positions of the complementaritydetermining regions (CDRs) that are responsible for the catalytic activity.

\section{Transition-state analogue approach}

The design of an antibody catalyst most often relies on the use of a hapten that is sterically and electronically similar to the transition state (or high-energy intermediate) of the reaction to be catalysed. The concept relies on the higher affinity of the catalyst for the transition state of the reaction compared to that for the substrate. It has been successfully applied to reactions in which the transition state differs considerably from the ground state and has been validated by the structures of esterolytic antibodies [3,5] and, recently, of antibodies catalysing porphyrin metallation, unimolecular cyclisations and pericyclic rearrangements (Table 1 ).

\section{Metal chelatase: catalysis by distortion}

Metal chelatase antibody 7G12 utilises binding energy to induce geometric strain in the porphyrin substrate 1 (Figure 1a) [7]. The antigen-binding site lies at the interface of the heavy and light chain variable domains $\left(\mathrm{V}_{\mathrm{H}}\right.$ and $\mathrm{V}_{\mathrm{L}}$, respectively). Binding of the distorted conformation of the transition-state analogue hapten 3 is carried out by hydrophobic packing interactions with the antibody (Figure 2a). This fixes the position of pyrrole ring B, whereas pyrrole ring A, which is not held in position by $\mathrm{V}_{\mathrm{H}}$, can be activated for distortion. Analysis of the sequence of the germline antibody, coupled to mutagenesis, shows that the somatic mutation at position H100c (serine to methionine) increases the affinity of the antibody for the distorted transition state and therefore suggests that MetH100c, which occupies the floor of the binding pocket, is a key residue in the strain mechanism of antibody $7 \mathrm{G} 12$. 
Table 1

Kinetic parameters and some structural characteristics of nonhydrolytic antibody catalysts*.

\begin{tabular}{|c|c|c|c|c|c|c|c|}
\hline \multirow[t]{2}{*}{ Antibody } & \multirow[t]{2}{*}{ Activity } & \multicolumn{3}{|c|}{ Kinetic parameters } & \multicolumn{3}{|c|}{ Structural features } \\
\hline & & $\mathrm{k}_{\mathrm{cat}} / \mathrm{K}_{\mathrm{m}}\left(\mathrm{M}^{-1} \mathrm{~min}^{-1}\right)$ & $\mathrm{k}_{\text {cat }}\left(\min ^{-1}\right)$ & $\mathrm{k}_{\text {cat }} / \mathrm{k}_{\text {non }}$ & Resolution $(\AA)$ & PDB code & References \\
\hline 7G12 & Porphyrin metallation ${ }^{\dagger}$ & 2.7 & 0.4 & 1700 & 2.4 & 3fct & [7] \\
\hline $5 \mathrm{C} 8$ & endo cyclisation of an epoxyalcohol* & $2.8 \times 10^{3}$ & 1.7 & ND\$ & 2.0 & $25 c 8$ & [8] \\
\hline 19A4 & Polyene cyclisation & 66 & 0.021 & $>2 \times 10^{3}$ & 2.7 & $1 \mathrm{cf8}$ & [10] \\
\hline $1 \mathrm{~F} 7$ & Chorismate mutase & $1.4 \times 10^{5}$ & 0.07 & 190 & 3.0 & 1 fig & [12] \\
\hline AZ-28 & oxy-Cope rearrangement & 311 & 0.023 & 5300 & 2.6 & 1 axs & [15] \\
\hline Germline & & $1.1 \times 10^{4}$ & 0.8 & $1.63 \times 10^{5}$ & 2.0 & $1 d 5 c$ & [16] \\
\hline $39-A 11$ & Diels-Alderase & $\begin{array}{l}3.5 \times 10^{4 \#} \\
5.4 \times 10^{4}\end{array}$ & 40.2 & $0.35 \mathrm{M}$ & 2.4 & $1 \mathrm{a} 4 \mathrm{k}$ & [17] \\
\hline Germline & & $\begin{array}{l}7.3 \times 10^{3 \#} \\
2.3 \times 10^{49}\end{array}$ & 10.2 & $0.09 \mathrm{M}$ & 2.1 & $1 \mathrm{a} 4 \mathrm{j}$ & [17] \\
\hline $1 \mathrm{E} 9$ & Diels-Alderase & $\begin{array}{c}5.4 \times 10^{3 \#} \\
450^{4}\end{array}$ & 13 & $10^{3} \mathrm{M}$ & 1.9 & $1 \mathrm{c} 1 \mathrm{e}$ & [19] \\
\hline 13G5 & exo Diels-Alderase & $\begin{array}{l}0.44^{\#} \\
0.12^{ף}\end{array}$ & $1.2 \times 10^{-3}$ & $6.9 \mathrm{M}$ & 1.95 & 1 a3l & [18] \\
\hline 28B4 & $\begin{array}{l}\text { Periodate-dependent } \\
\text { oxidation of sulfide }\end{array}$ & $1.1 \times 10^{7}$ & 492 & $9.65 \mathrm{M}$ & 1.9 & $1 \mathrm{kel}$ & [22] \\
\hline $33 \mathrm{~F} 12 * *$ & $\begin{array}{l}\text { Aldolase } \\
\text { Allylic rearrangement }\end{array}$ & 230 & $\begin{array}{c}10^{-3}-5 \\
0.54\end{array}$ & $\begin{array}{l}10^{5}-10^{7} \\
1.2 \times 10^{3}\end{array}$ & 2.15 & $1 \mathrm{axt}$ & [25] \\
\hline 4B2 & $\begin{array}{l}\text { Allylic rearrangement } \\
\text { Kemp elimination }\end{array}$ & $\begin{array}{l}1.2 \\
1.3\end{array}$ & $\begin{array}{c}1.6 \times 10^{-3} \\
630\end{array}$ & $\begin{array}{c}1500 \\
6.3 \times 10^{3}\end{array}$ & 1.87 & $1 f 3 d$ & [30] \\
\hline
\end{tabular}

*A previously published review [4] covers the chorismate mutase and the text (see also Update). †The kinetic constants are for copper sulfide oxidase antibody catalysts, which are only briefly discussed in

metallation. \$Disfavoured reaction. §Not determined: the disfavoured endo product is negligible in the uncatalysed reaction. \#Diene. IDienophile. **Unliganded structure.

an $N$-oxide analogue of hapten 6 , in which the $N$-oxide oxygen makes an hydrogen bond to AspH95 [8].

be essential for catalytic activity; its carboxylate is positioned $1.9 \AA$ from the centre of the porphyrin ring. This indicates that it could facilitate metal complexation either by acting as a general base to deprotonate the nitrogen of the porphyrin substrate or by forming a metal-oxygen bond. This would explain that the catalytic efficiency of 7 G12 approaches that of the enzyme ferrochelatase.

\section{Unimolecular cyclisation}

Antibodies that catalyse unimolecular cyclisation use binding energy to constrain the acyclic substrate, leading to an appropriate conformation for concerted cyclisation. Antibody 5C8 catalyses the one-step 6-endo cyclisation of epoxyalcohol 4, a reaction that is strongly disfavoured in solution, with high regioselectivity and enantioselectivity (Figure 1b) [8]. The catalytic effect appears to come from electronic stabilisation and, possibly, general acid/base catalysis. The antibody selectively stabilises the higher energy six-membered transition state by electrostatic stabilisation of the higher partial positive charge that develops at the epoxide carbon atom. Although some charge stabilisation may be achieved by the distant residue AspH101, the main charge stabilisation probably comes from cation $-\pi$ interactions, as numerous aromatic or histidine residues border the antibody combining site (Figure 2b). In addition, HisL89 and AspH95 could act as a base/acid dyad, respectively, to deprotonate the alcohol group and increase its nucleophilicity, or to protonate the epoxide oxygen atom in order to make it a better leaving group and facilitate epoxide opening. This interpretation is supported by the structure of antibody $5 \mathrm{C} 8$ complexed with
Polyene cyclisation is another class of unimolecular rearrangement reaction; it is among the most demanding reactions to have been catalysed by antibodies and is of great interest to gain access to steroidal carbon frameworks. Although the uncatalysed cationic reactions result in a mixture of elimination and addition products, they can be re-routed via antibody catalysis to yield predominantly one product [9]. Antibody 19A4 catalyses a multistep cationic cyclisation (Figure 1c) [10]. After binding the linear polyene substrate 7 in a productive chair-chair conformation, it has been proposed that the carbocation cyclisation cascade is initiated by AsnH35, which might help trigger departure of the sulfonate leaving group by hydrogen bonding (Figure 2c). This assumes that the sulfonate leaving group would occupy the same position as the nitroxide oxygen of hapten 9; this needs further crystallographic evidence. The antibody mainly utilises aromatic amino acid residues for the stabilisation of either the positive charge developing on the substrate $\mathrm{C} 1$ atom or the C5 and C9 high-energy carbocationic intermediates. These cation $-\pi$ interactions control the stereochemistry of the two-step cyclisation, as indicated by the formation of only the trans decaline skeleton 8 , but there is no catalytic base to direct the regiochemistry of the final proton elimination step to yield one exclusive olefin.

\section{Unimolecular pericyclic rearrangements}

The transition-state analogue approach has been particularly useful as a source of catalysts for transformations such 
Figure 1

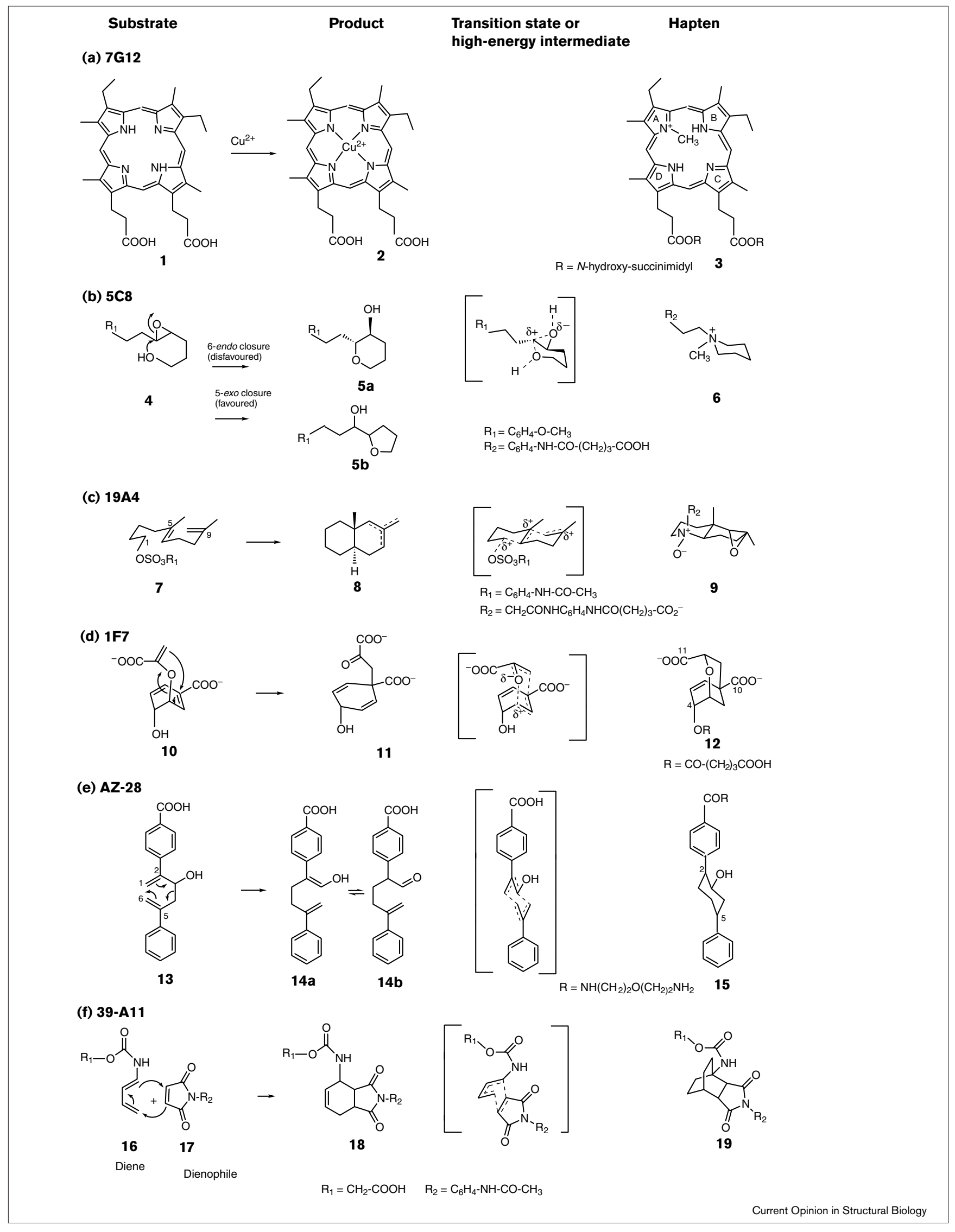


(g) $1 \mathrm{Eg}$

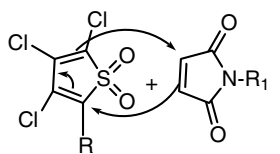

20

Diene Dienophile<smiles></smiles>

22

(h) 13G5

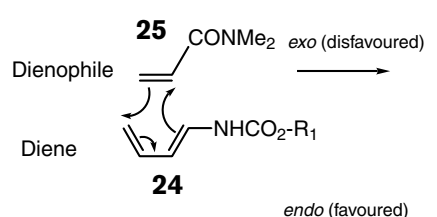

endo (favoured)

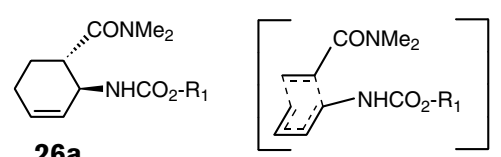

26a

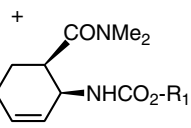

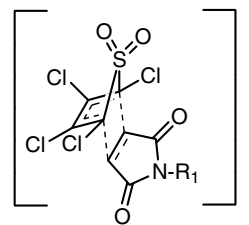

$\mathrm{R}=\mathrm{CH}_{2}-\mathrm{O}-\mathrm{CH}_{2}-\mathrm{CO}_{2}^{-}$

$\mathrm{R}_{1}=\mathrm{CH}_{2}-\mathrm{CH}_{3}$

$\mathrm{R}_{2}=\left(\mathrm{CH}_{2}\right)_{5}-\mathrm{CO}_{2}^{-}$

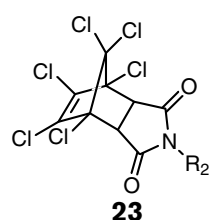

23

26b

(i) 28B4<smiles>CSCc1ccc([N+](=O)[O-])cc1</smiles>

28

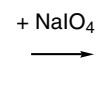<smiles>C[SH](O)Cc1ccc([N+](=O)[O-])cc1</smiles>

29

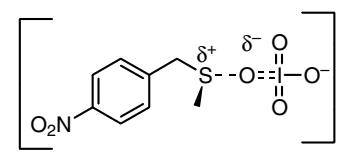

$\mathrm{R}_{1}=$ 4-carboxybenzy $\left.\mathrm{R}_{1}=4-\mathrm{carb} \mathrm{C}_{2}\right)_{3}-\mathrm{COOH}$
$\mathrm{R}_{2}$

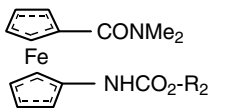

27a

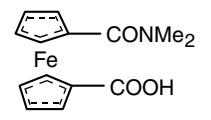

27b

$+\mathrm{NaIO}_{3}$<smiles>O=C(O)CCCC[NH+](Cc1ccc([N+](=O)[O-])cc1)CP(=O)([O-])[O-]</smiles>

(j) 33F12

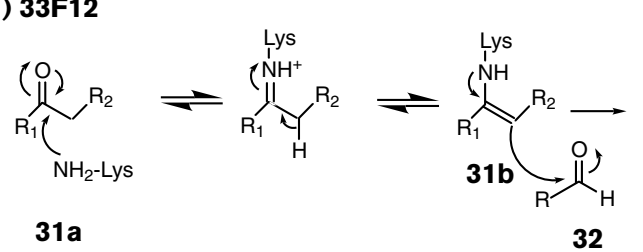<smiles>[2H]C(O)C([2H])C([2H])[18OH]</smiles><smiles>[R]C(=O)CC(C)=O</smiles>

33a

33b 34

$\mathrm{R}_{4}=\left(\mathrm{CH}_{2}\right)_{2}-\mathrm{C}_{6} \mathrm{H}_{4}-\mathrm{NHCO}-\left(\mathrm{CH}_{2}\right)_{3}-\mathrm{COOH}$

(k) 4B2

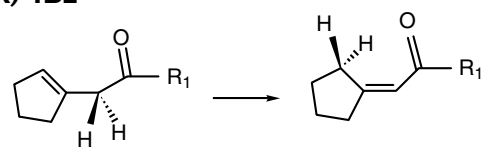

35
36

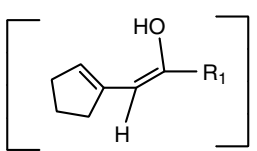

$\mathrm{R}_{1}=\mathrm{C}_{6} \mathrm{H}_{4}-\mathrm{NH}-\mathrm{CO}-\mathrm{CH}_{3}$

$\mathrm{R}_{2}=\mathrm{C}_{6} \mathrm{H}_{4}-\mathrm{NH}-\mathrm{CO}-\left(\mathrm{CH}_{2}\right)_{3}-\mathrm{COOH}$

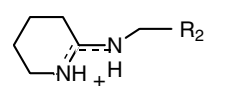

37

Current Opinion in Structural Biology as cycloadditions, which are characterised by a highly ordered transition state. As there are relatively few examples of enzyme-catalysed pericyclic processes and in view of their synthetic utility [11], these reactions remain a major focus for the catalytic antibody field, as illustrated by new structural reports documenting antibody-catalysed pericyclic reactions (Table 1).

Unimolecular pericyclic rearrangements involve a conformationally flexible substrate, which evolves towards a chair-like transition state in which the $4 \pi$ and $2 \sigma$ orbitals partially overlap in a cyclic array. The first structure of a complex between an antibody catalyst and its hapten involved the chorismate mutase antibody 1F7 (Figure 1d) [12]. The Claisen rearrangement is a well-suited goal for antibody catalysis, as the enzyme-catalysed reaction does not require any functional group to form a covalent intermediate with the substrate or to participate in general acid/base catalysis [13,14]. Antibody 1F7 organises the substrate into the chair-like reactive conformation by forming van der Waals interactions and a network of hydrogen bonds with the carboxylates. The natural 
Schemes of the reactions catalysed by the structurally characterised antibodies. (a) 7G12 catalyses metal insertion into the mesoporphyrin ring of 1 . As the enzyme-catalysed reaction is thought to result from strain of the planar porphyrin ring substrate toward a distorted transition state, this antibody was induced by $\mathrm{N}$-alkylmesoporphyrin hapten $\mathbf{3}$, which adopts a distorted geometry mimicking that of the transition state. (b) $5 \mathrm{C} 8$ catalyses the disfavoured intramolecular 6-endo ring closure of trans-epoxyalcoho 4 to yield the six-membered product $\mathbf{5 a}$, whereas the uncatalysed reaction gives the five-membered product 5 b. 5 C8 was generated against $N$-methyl piperidinium hapten $\mathbf{6}$, which possesses both a ring conformation that approximates the required geometry during 6 -endo ring closure and a positive charge mimicking that which develops at the epoxide carbon atom during the transition state. No feature of the hapten mimics the developing negative charge at the epoxide oxygen atom. (c) 19A4 catalyses the cationic cyclisation of polyene $\mathbf{7}$ to form the bicyclic isomeric products $\mathbf{8}$. The half-chair conformation of the second six-membered ring in hapten $\mathbf{9}$ was expected to induce an antibody combining site that enforces the appropriate conformation of substrate 7 . The positively charged nitrogen atom of $\mathrm{N}$-oxide hapten $\mathbf{9}$ mimics the developing positive charge on $\mathrm{C} 1$ of substrate 7. (d) $1 \mathrm{~F} 7$ catalyses the unimolecular Claisen rearrangement of chorismate 10 to prephenate 11. It was induced against hapten 12, which mimics the chair-like geometry, but not the highly dipolar nature, of the transition state. (e) AZ-28 catalyses the oxy-Cope rearrangement of hexadiene 13. Rapid conversion of the enol group in product $14 \mathrm{a}$ leads to the aldehyde $\mathbf{1 4 b}$, which is trapped in situ with hydroxylamine. AZ-28 was obtained through immunisation with hapten 15, a chair-like analogue of the preferred chair transition state of the rearrangement. (f) 39-A11 catalyses the Diels-Alder reaction of diene 16 and dienophile 17 to form bicyclic product 18. 39-A11 was generated against bicyclic hapten 19 , a mimic of the boat-like transition state of the reaction. The conformation of hapten 19 differs from that of the chair-like product, thereby preventing product inhibition. (g) The product of the cycloaddition of diene $\mathbf{2 0}$ and dienophile $\mathrm{N}$-ethylmaleimide 21 , catalysed by $1 \mathrm{E} 9$, spontaneously extrudes $\mathrm{SO}_{2}$ and oxidises to form aromatic product 22, which is sufficiently different from the transition state that no product inhibition occurs. (h) $13 \mathrm{G} 5$ catalyse the Diels-Alder reaction between carbamate diene 24 and $\mathrm{N}, \mathrm{N}$-dimethylacrylamide $\mathbf{2 5}$ to give only the exo regioisomer $\mathbf{2 6 a}$ in high enantiomeric excess. This reaction is disfavoured relative to the formation of the endo adduct $\mathbf{2 6} \mathbf{b}$. 13G5 was raised against ferrocene derivative $27 \mathrm{a}$, which, with its two pentagonal delocalised $\pi$-electron ring systems stacked upon each other, acts as a loose mimic of a very early boat-like transition state. 13G5 was crystallised in the presence of the more chemically stable ferrocene inhibitor $\mathbf{2 7} \mathrm{b}$. The ferrocenyl carboxylic acid of $\mathbf{2 7 b}$ represents the carbamate of diene $\mathbf{2 4}$ and the ferrocenyl (dimethylamino)-carbonyl mimics the N,N dimethylacrylamide of dienophile 25. (i) $28 \mathrm{~B} 4$ catalyses the oxidation of sulfide 28 with periodate to form sulfoxide 29. The ammonium and phosphonate of hapten 30 , respectively, mimic the positive and negative charges that develop on sulfur and periodate in the transition state. (j) 33F12 catalyses a large array of aldol condensations. The Schiff base formed between an active site lysine residue and various ketone substrates $\mathbf{3 1 a}$ leads to the enamine $\mathbf{3 1 b}$. Addition of this enamine to different aldehydes 32 leads to the Schiff base 33a, which is hydrolysed to give the $\beta$-hydroxyketone product $33 \mathrm{~b}$. During the immunisation process, diketone hapten $\mathbf{3 4}$ can form a stable vinylogous amide by reaction with the reactive lysine sidechain. (k) 4B2 catalyses the isomerisation of $\beta-\gamma$ unsaturated ketone 35 to $\alpha-\beta$ unsaturated ketone 36 . 4B2 was induced against hapten $\mathbf{3 7}$, which possesses an amidinium function for the induction of a complementary charged residue in the antibody, at a location that allows it to act as a general base. enzymes accelerate the rearrangement more efficiently because they provide more hydrogen-bonding and direct electrostatic interactions to constrain the conformation of the chorismate substrate. In addition, they destabilise the substrate by hydrogen bonding to the hydroxyl group and, unlike the antibody, possess a dipolar active site well suited to stabilise the dipolar transition state.

Antibody AZ-28 catalyses a related [3,3] sigmatropic rearrangement that has no natural counterpart, the oxyCope rearrangement of hexadiene 13, through a combination of electronic and entropic effects (Figures 1e and 2d) [15]. Binding of the substrate in a chair-like conformation places the hexadiene in favourable orbital alignment. However, the two phenyl substituents of hapten 15 are rotated out of plane with respect to the cyclohexyl ring. This conformation of the transition state is catalytically unfavourable because of decreased $\pi$ orbital overlap between the hexadiene system and the aryl substituents. A comparison of the X-ray structures of the germline and affinity-matured antibodies [16] has shown that the somatic mutation of SerL34 to AsnL34 decreases the flexibility of CDR loop H3 and leads to restricted substrate rotation around the $\mathrm{C} 2$ aryl bond and decreased orbital overlap. This explains why the rate enhancement of the mature antibody is, surprisingly, 30-fold lower than that of its germline precursor.

\section{Diels-Alderases: bimolecular pericyclic reactions}

The crystal structures of three antibody catalysts that accelerate a Diels-Alder reaction have been recently reported (Table 1) [17-19]. This reaction consists of the 1,4 cycloaddition of an alcene (or dienophile) to a diene, and yields cyclic compounds of great interest in synthetic organic chemistry. It is facilitated by the presence of electron-donating groups on the diene (donor) and electron-withdrawing functionalities on the dienophile (acceptor), and involves the stacking of the $4 \pi$ and $2 \pi$ orbitals to form a boat-shaped transition state. Two approaches have been used to generate antibodies capable of catalysing this reaction. The first uses rigid, bicyclic transition-state analogues 19 and 23 (Figure 1f,g), and the other uses a conformationally unrestricted ferrocene derivative, 27a (Figure 1h).

The structure of 39-A11 suggests that the antibody binds the diene 16 and dienophile 17 in a reactive orientation and reduces their degrees of freedom (Figure 2e) [17]. Structural and functional studies of the germline precursor of 39-A11 suggest that a single somatic mutation, SerL91Val, is largely responsible for the 40-fold and fourfold increases in binding affinity and catalytic activity, respectively, of the mature antibody, probably because it partially eliminates a prominent cavity present around residues L91 and L89. The still low catalytic efficiency 


\section{Figure 2}

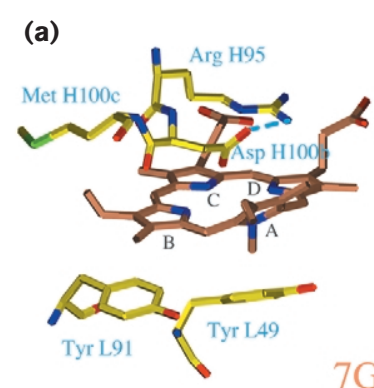

(c)

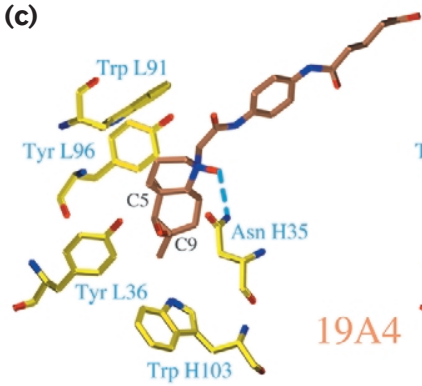

(e)

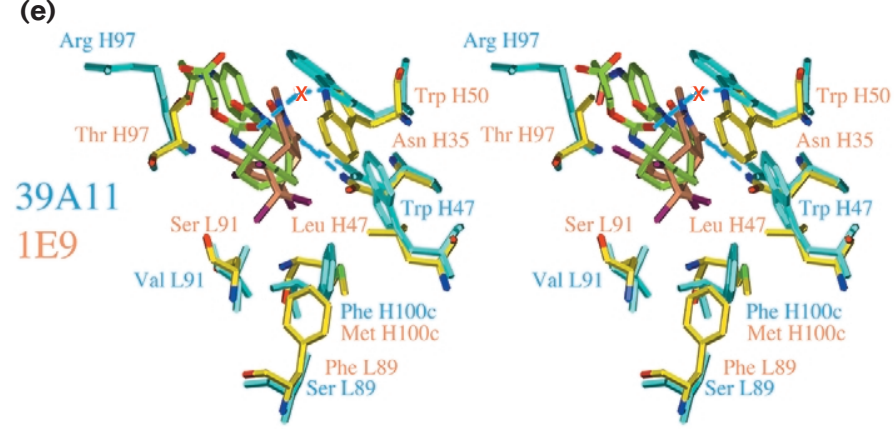

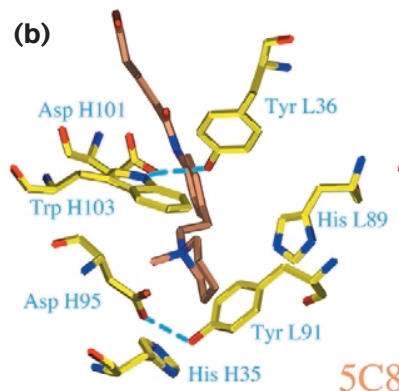
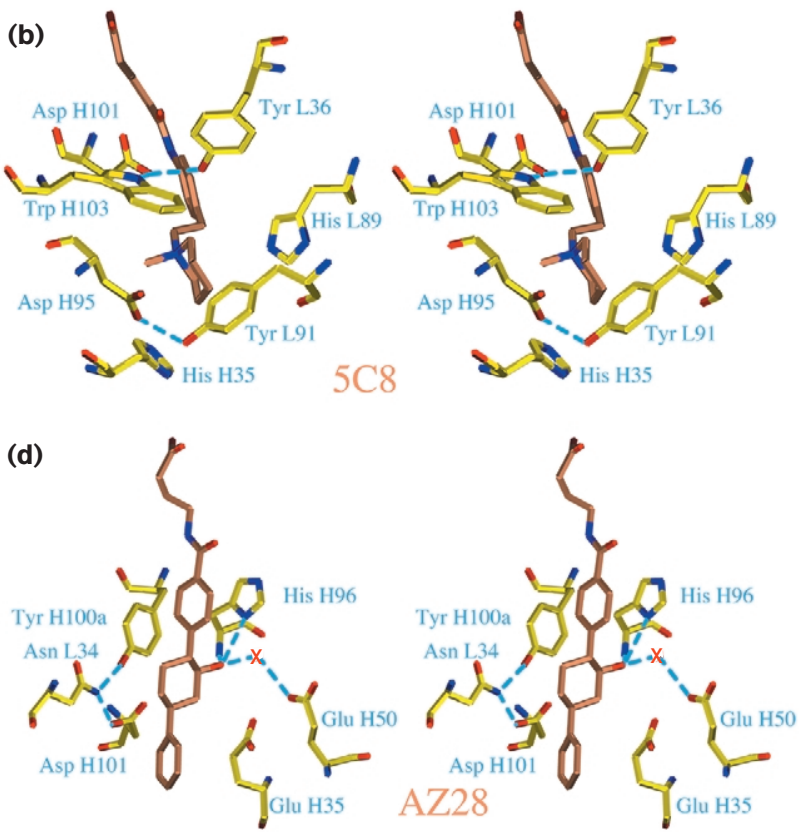

(f)
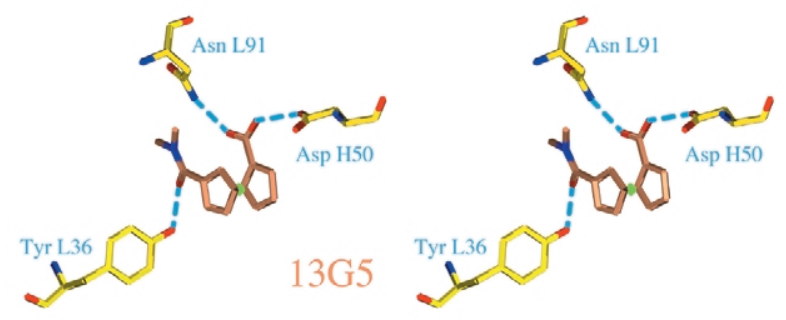

(h)
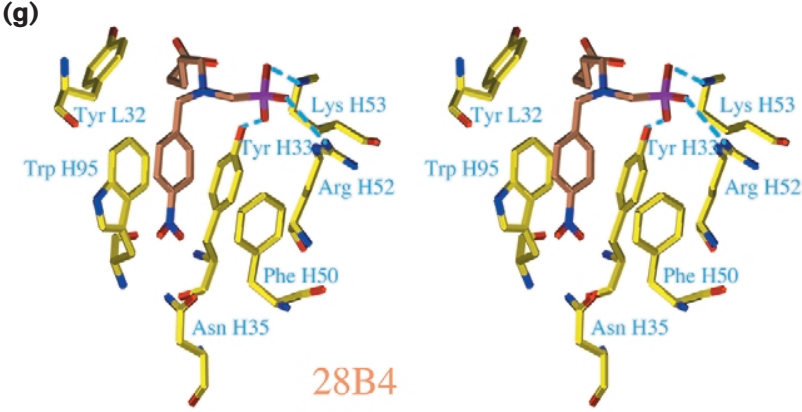

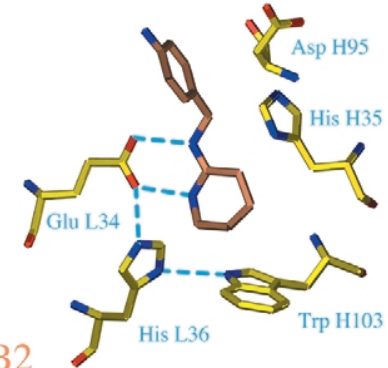

Current Opinion in Structural Biology of 39-A11 can be attributed to the loose fit of its transition state with the binding site, which is exacerbated by the incorporation of an ethano bridge in the hapten 19 that occupies the bottom of the cavity and has no counterpart in the transition state (Figure 1f).

The crystal structure of 39-A11 has been compared to that of the related antibody $1 \mathrm{E} 9$, the most efficient Diels-Alder antibody catalyst known, which possesses a
CDR H3 loop from a different origin, but of the same length and conformation (Figure 2e) [19]. Although the two antibodies stabilise the transition state using the same hydrogen bond between AsnH35 and the dienophile carbonyl, the higher efficiency of 1E9 comes from its almost perfect shape complementarity with its transition-state analogue hapten 23 (Figure $1 \mathrm{~g}$ ), which is mostly due to two somatic mutations. Although germline residue SerL91 is not mutated in $1 \mathrm{E} 9$, the nearby cavity that is 
Antibody-hapten complexes, showing the key antibody-ligand interactions that explain the catalytic activity. (a) 7G12. The antibody binds one isomer of porphyrin $\mathbf{3}$, with pyrrole ring $\mathrm{A} \mathrm{N}$-methylated. Pyrrole rings $A$ and $B$ are buried at the bottom of the pocket, whereas pyrrole rings $C$ and $D$, which bear the propionate substituents, are exposed to the solvent. Pyrrole rings $B, C$ and $D$ are coplanar and pyrrole ring $A$ is distorted out of this plane by $42^{\circ}$. On one face of the porphyrin, TyrL49 and TyrL91 $\pi$-stack on pyrrole rings $A$ and $B$, respectively, whereas on the other side, ArgH95, AspH100b and MetH100c from CDR H3 pack tightly against pyrrole rings $\mathrm{B}, \mathrm{C}$ and D. The position of catalytic AspH100b is fixed by a hydrogen bond to ArgH95. (b) 5C8. The ammonium positive charge of hapten 6 is stabilised by cation $-\pi$ interactions with TyrL91, HisL89, HisH35, $\mathrm{TrpH} 103$ and TyrL36, and by a weak electrostatic interaction with AspH101, which hydrogen bonds to TrpH103 and TyrL36. HisL89 is a putative base that deprotonates the alcohol oxygen of 4 and AspH95, which hydrogen bonds to TyrL91, is a putative acid that protonates its epoxide. The benzene moiety is positioned by $\pi$-stacking and hydrophobic interactions with HisL34 and ProH96, respectively (not shown). (c) 19A4. The $N$-oxide oxygen of hapten 9 hydrogen bonds to Asn $\mathrm{H} 35$ and its positively charged nitrogen is stabilised by cation $-\pi$ interactions with TrpL91 and TyrL96. TrpL91 and TyrL36 probably stabilise the C5 carbocation intermediate and TyrL36 and TrpH103 probably stabilise the $\mathrm{C} 9$ carbocation intermediate. (d) AZ-28. AsnL34, which is positioned by hydrogen bonds to $\mathrm{AspH} 101$ and $\mathrm{TyrH} 100 \mathrm{a}$, restricts rotation around the $\mathrm{C} 2$ and $\mathrm{C} 5$ aryl bonds. The hydrogen bonds between residue L34 and $\mathrm{H} 100 \mathrm{a}$ are $2.6 \AA$ and $3.5 \AA$ in the mature antibody-hapten and the germline antibody-hapten complexes, respectively. The 2-phenyl substituent $\pi$-stacks with TyrL91 (not shown), HisH96 and TyrH100a. The hydrogen bonds between the hydroxyl substituent and either residue HisH96 or the bridging water to GluH50 may enhance the rate of rearrangement by increasing the electron density on the hydroxyl substituent of the substrate. (e) Superposition of 39-A11 and 1E9. Water molecules are indicated as red crosses, hydrogen bonds as blue dashed lines. The atom colouring scheme is as follows: carbon atoms of the proteins in yellow (except for 39-A11 in cyan), carbon atoms of the ligand in orange (except for 39-A11 in spring green), oxygen in red, nitrogen in blue, sulfur in green, phosphorus in magenta, chloride in maroon. Although TrpH50 adopts different conformations in each antibody, it $\pi$-stacks, in both cases, with the succidimyl group of the hapten, which is isosteric with the maleimide ring of the dienophile substrate. In addition, the dienophile is fixed in close proximity to the diene through $\mathrm{AsnH} 35$, which donates a hydrogen bond to one of the succinimide carbonyl groups of haptens 19 or 23 , and, in 39-A11, through hydrophobic interactions between $\mathrm{ArgH} 97$ and the phenyl substituent of the hapten. The diene is oriented, in 39-A11, by a water-mediated hydrogen bond between its carbamate substituent and $\mathrm{TrpH} 50$, and, in 1E9, by tight contacts to the antibody, due to mutations of residues in positions $\mathrm{L} 89, \mathrm{H} 47$ and H100c. (f) 13G5. In the 13G5-27b complex, TyrL36, which is oriented by a hydrogen bond with the amide of GlyH100c (not shown), accepts a hydrogen bond from the amide carbonyl oxygen of the pentadienyl that mimics the dienophile. AsnL91 and AspH50, which are positioned by hydrogen bonds to HisL32 and TrpH47, respectively (not shown), hydrogen bond to the carboxylic acid that is equivalent of the carbamate of the diene. (g) 28B4. TyrL32 stabilises the positive charge of the ammonium of hapten 30 by a cation $-\pi$ interaction. TyrH33, ArgH52 and LysH53 contact the oxygens of the phosphonate via hydrogen bonds and salt bridges. The phenyl ring of hapten $\mathbf{3 0}$ is sandwiched between the aromatic rings of TrpH95 and PheH50. (h) 4B2. GluL34, which is positioned by a hydrogen-bond network with HisL36 and TrpH103, makes an ionic interaction with the bidentate amidinium moiety of hapten 37 . GluL34 acts as a general base that abstracts the allylic proton. HisH35, which points its protonated nitrogen towards the hapten, or AspH95, after a conformational change, might act as an acid to protonate the carbonyl substrate or the enediolate intermediate. present in the 39-A11 complex is filled in by residue L89, which is mutated from a serine to a phenylalanine in $1 \mathrm{E} 9$. The second mutation is the rare substitution of framework residue $\mathrm{TrpH} 47$ by leucine, which significantly deepens the active site by allowing the bulky contacting residue TrpH50 to rotate its indole ring and fill in the vacated space. In addition, residue $\mathrm{H} 100 \mathrm{c}$, which provides the hydrophobic floor of the binding pockets, is a phenylalanine in $39-\mathrm{A} 11$, but a methionine in $1 \mathrm{E} 9$, which is much more adaptable to the ligand shape because of the flexibility of its sidechain. Finally, the 1E9 transition state is less polar than that of $39-\mathrm{A} 11$, which catalyses a reaction involving a strong donor diene 16 and acceptor dienophile 17 (Figure 1f,g), and is better stabilised in the relatively nonpolar environment of the $1 \mathrm{E} 9$ binding site.

Antibody 13G5, which catalyses a disfavoured exo Diels-Alder reaction, was raised against transition-state analogue 27a, a ferrocene derivative with cyclopentadienyl rings that are able to rotate freely in solution (Figure $1 \mathrm{~h}$ ) [18]. In the inhibitor (27b)-antibody complex, the ferrocene ring rotation is restricted because of steric constraints and specific interactions with the antibody. This validates the idea that the immune system can select one conformer of a flexible hapten that mimics one of the Diels-Alder transition states and, hence, can produce a specific Diels-Alderase. From the three hydrogen bonds that are made between $27 \mathrm{~b}$ and the antibody, a catalytic mechanism was proposed (Figure 2f). TyrL36 acts as a Lewis acid, activating the dienophile for nucleophilic attack. AspH50 would act as an electron donor that increases the nucleophilicity of the diene by hydrogen bonding to the NH of the diene carbamate. A third hydrogen bond, which also contributes to orient the substrates and control product formation and stereoselectivity, occurs between AsnL91 and the carbonyl oxygen of the carbamate. The three key residues have their sidechains fixed by an extensive hydrogen-bond network.

Antibodies that catalyse pericyclic reactions were designed to perform as an entropy trap and X-ray structures of Diels-Alderase antibody catalysts show how the catalyst controls the orientation of the two substrates to form the highly ordered transition state. However, the activation parameters of the two unimolecular rearrangements reveal that these antibodies function primarily by reducing the enthalpy of activation of the reaction [20,21]. The catalytic activity of antibodies catalysing pericyclic rearrangements can thus be explained partly by the presence of a hydrophobic microenvironment that serves to sequester the substrate(s) from aqueous solution and preorganise it (them) into a reactive complex, through a myriad of van der 


\section{Figure 3}

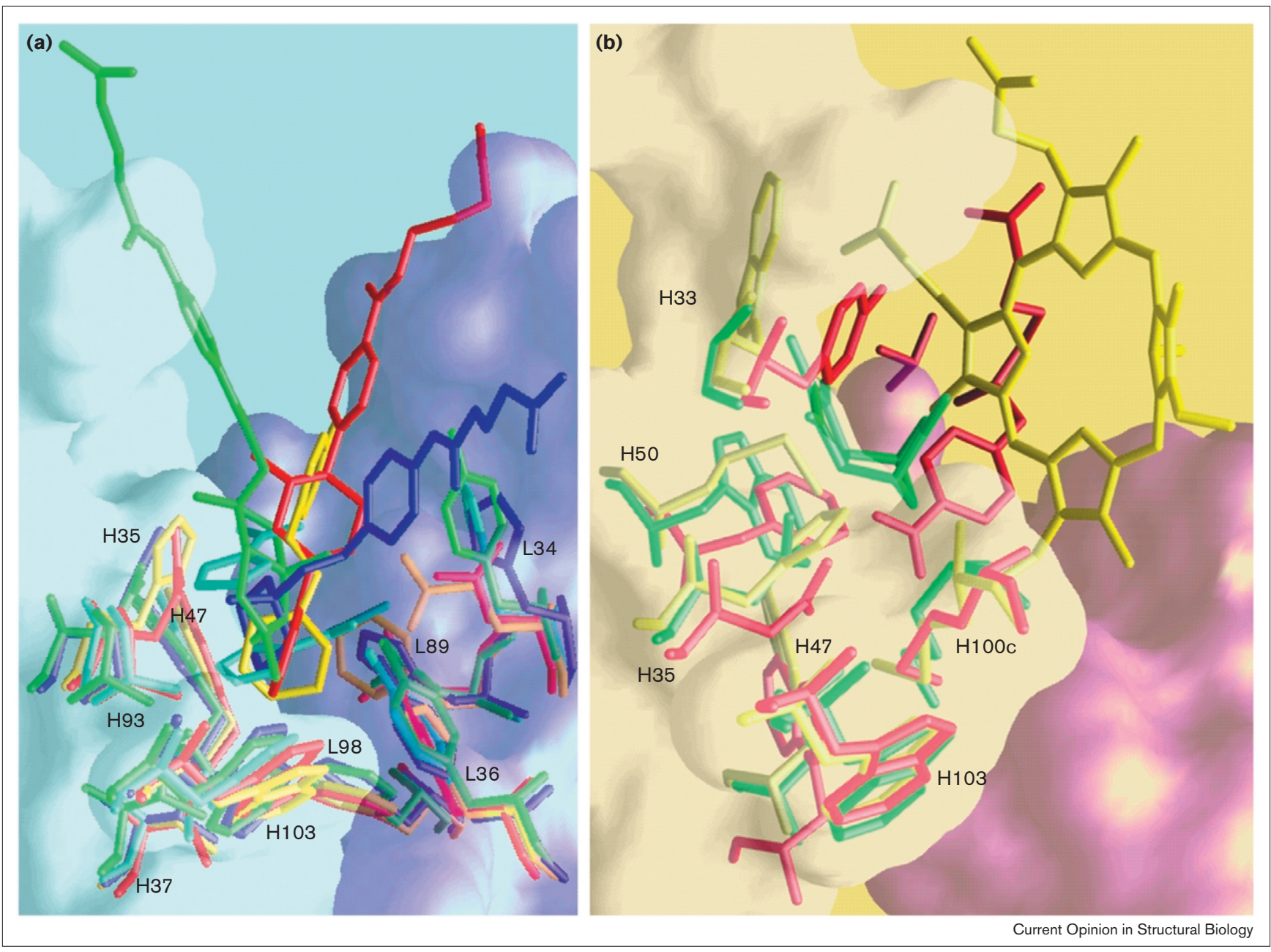

Comparison of the cavity shapes of the nonhydrolytic catalytic antibodies. (a) The cavity of antibodies that possess a CDR H3 loop with a small residue at position $\mathrm{H} 100 \mathrm{c}$. A section of the molecular surface of the combining site of antibody 4B2, omitting CDR H3 (in front), coloured in light blue for the heavy chain and light purple for the light chain, was obtained with the program GRASP. The $\alpha$ carbons of residues of the framework ( $L 1-L 25, L 33-L 49$, L53-L90, L97-L107, H1-H25, H36-H51, H57-H93 and $\mathrm{H} 103-\mathrm{H} 111)$ of antibodies 5C8, 19A4, AZ-28 and 13G5 have been superimposed on those of $4 \mathrm{~B} 2$. The root mean squared deviations from 4B2 are 1.031 $\AA$ (5C8), $1.545 \AA$ (19A4), $0.760 \AA$ (AZ-28) and $1.072 \AA$ (13G5). The hapten structures and the common residues of the combining site (L34, L36, L89, L98, H35, H37, H47, H93 and $\mathrm{H} 103)$ are shown in stick representation, coloured in yellow (4B2), dark blue (5C8), green (19A4), red (AZ-28) and cyan (13G5). The hydrolytic antibodies CNJ206, 48G7 and 17E8, which possess, respectively, glycine, glycine and valine at position $\mathrm{H} 100 \mathrm{c}$, and $\mathrm{D} 23$, which has an extended conformation of its $\mathrm{H} 3 \mathrm{C}$-terminal region that removes MetH100c from the bottom of the antigen-binding site, share the same combining site illustrated here [3,5,31]. The aryl (AZ-28, esterases), pentadienyl (13G5) or hydrophobic ring (5C8, 4B2, 19A4) substituents of the haptens maximise hydrophobic contacts with the antibody at the hydrophobic bottom of the cavity, which is made of conserved residues of the framework (TrpH103, PheL98, H93, TrpH47, Val/IleH37). This leads to a common shape for the bottom of the combining sites. The more variable residues in positions
L34, L36, L89, H35 and L91 (not shown, for clarity) form the walls of the cavity and most often participate in catalysis. The terminal part of the haptens, which is linked to the carrier protein during the immunisation process, is solvent accessible and oriented differently in the various structures. (b) The cavity of antibody 7G12, as an example of an antibody with a bulky residue at position $\mathrm{H} 100 \mathrm{c}$ of CDR H3. A section of the molecular surface of the combining site of 7G12 (omitting $\mathrm{H} 95$ and $\mathrm{H} 100 \mathrm{~b}$, in front), coloured in light yellow for the heavy chain and pink for the light chain, is shown in the same orientation as (a). The $\alpha$ carbons of residues of the framework of antibodies 7G12, 1E9 and 28B4 have been superimposed on those of 4B2 [shown in (a)]. The root mean squared deviations are $1.044 \AA$ (7G12), $1.476 \AA$ (28B4) and $0.857 \AA$ (1E9). The hapten structures and some common residues of the combining site $(\mathrm{H} 33, \mathrm{H} 50$, $\mathrm{H} 100 \mathrm{c}$ ), as well as $\mathrm{H} 35, \mathrm{H} 47$ and $\mathrm{TrpH} 103$, are shown in stick representation, coloured in yellow (7G12), green (1E9) and red (28B4). The atypical combining sites of hydrolytic antibodies 7C8 and 6D9 [compared with the cavity illustrated in (a)] [32] can be linked to the presence of a bulge phenylalanine at position $\mathrm{H} 100 \mathrm{c}$ in both cases. Residue $\mathrm{H100c}$, in each antibody, fills in the bottom of the cavity seen in (a), preventing contact of the haptens with buried $\mathrm{TrpH} 103$. The ligands bind at the upper part of the $\mathrm{V}_{L}-\mathrm{V}_{\mathrm{H}}$ interface and contact almost exclusively residues of the CDRs. There are, therefore, fewer common residues that make up the combining site ( $\mathrm{H33}, \mathrm{H} 50, \mathrm{H} 95, \mathrm{H} 100 \mathrm{a}$ and $\mathrm{H} 100 \mathrm{c}$ ) and the cavity has different shapes and may be narrow, as illustrated here for 7G12. 
Table 2

Sequence comparison of the active site residues of the structurally characterised nonhydrolytic catalytic antibodies*.

\begin{tabular}{|c|c|c|c|c|c|c|c|c|c|c|c|c|c|}
\hline $\begin{array}{l}\text { (a) } \\
\text { Residue }\end{array}$ & Location & AZ-28 & $13 G 5$ & $5 C 8$ & $19 A 4$ & 4B2 & $\begin{array}{c}\text { (b) } \\
\text { Residue }\end{array}$ & Location & $1 \mathrm{~F} 7$ & 39A11 & $1 \mathrm{E} 9$ & 7G12 & 28B4 \\
\hline L32 & CDR1 & Tyr & His & Asn & Tyr & Tyr & L32 & CDR1 & Tyr & Tyr & Tyr & Pro & Tyr \\
\hline L34 & CDR1 & Asn ${ }^{\dagger}$ & Tyr & His & Tyr & Glu & L34 & CDR1 & His & His & His & Ala & Glu \\
\hline L36 & FR2 & Phe & Tyr & Tyr & Tyr & His & L36 & FR2 & Tyr & Tyr & Tyr & Tyr & Tyr \\
\hline L49 & FR2 & Tyr & Tyr & Tyr & Tyr & Tyr & L49 & FR2 & Tyr & Tyr & Tyr & Tyr & Tyr \\
\hline L89 & CDR3 & Leu & Ala & His & Leu & Phe & L89 & CDR3 & Gln & Ser & Phe & Gln & Phe \\
\hline L96 & CDR3 & Tyr & Tyr & Tyr & Tyr & Tyr & L94 & CDR3 & Tyr & Val & Phe & Tyr & Val \\
\hline L98 & FR4 & Phe & Phe & Phe & Phe & Phe & L96 & CDR3 & Leu & Pro & Pro & Leu & Arg \\
\hline Н33 & CDR1 & Trp & Trp & Tyr & Ala & Asn & L98 & FR4 & Phe & Phe & Phe & Phe & Phe \\
\hline H35 & CDR1 & Glu & Gly & His & Asn & His & НЗ3 & CDR1 & Asn & Gly & Gly & Trp & Tyr \\
\hline Н37 & FR2 & Val & Val & Val & Ile & Val & Н35 & CDR1 & Asn & Asn & Asn & His & Asn \\
\hline $\mathrm{H} 47$ & FR2 & Trp & Trp & Trp & Trp & Trp & Н37 & FR2 & Ile & Val & Val & Val & Ala \\
\hline H50 & FR2 & Glu & Asp & Gln & Tyr & Tyr & $\mathrm{H} 47$ & FR2 & $\operatorname{Trp}$ & Trp & Leu & Trp & Trp \\
\hline H93 & FR3 & Ala & Ala & Ala & Ala & Ala & $\mathrm{H} 50$ & FR2 & Asn & Trp & Trp & Met & Phe \\
\hline H96 & CDR3 & His & Gly & Pro & Tyr & Tyr & H53 & CDR2 & Tyr & Tyr & Tyr & Asn & Lys \\
\hline H98 & CDR3 & Tyr & Tyr & Tyr & - & - & H93 & FR3 & Ala & Val & Ala & Thr & Ala \\
\hline $\mathrm{H} 100 \mathrm{a}$ & CDR3 & Tyr & Thr & Gly & Gly & - & H95 & CDR3 & Arg & Ala & Gly & Arg & Trp \\
\hline $\mathrm{H} 100 \mathrm{c}$ & CDR3 & Gly & Gly & Gly & Ser & Gly & H96 & CDR3 & Arg & Glu & Thr & - & Gly \\
\hline $\mathrm{H} 101$ & CDR3 & Asp & Asp & Asp & Asp & Val & $\mathrm{H} 97$ & CDR3 & Asp & Arg & Thr & - & Ser \\
\hline \multirow[t]{4}{*}{$\mathrm{H} 103$} & FR4 & Trp & Trp & Trp & Trp & Trp & H98 & CDR3 & Gly & Leu & Ile & - & - \\
\hline & & & & & & & $\mathrm{H} 100 \mathrm{a}$ & CDR3 & Tyr & Arg & Arg & - & Tyr \\
\hline & & & & & & & H100b & CDR3 & Gly & Thr & Ala & Asp & Ala \\
\hline & & & & & & & $\mathrm{H} 100 \mathrm{c}$ & CDR3 & Phe & Phe & Met & Met & Met \\
\hline
\end{tabular}

*Antibodies with a small residue at position $\mathrm{H} 100 \mathrm{c}$, indicated in Table 2a (left), share a binding pocket, as illustrated in Figure 3a. Antibodies with methionine or phenylalanine at position $\mathrm{H} 100 \mathrm{c}$ indicated in Table $2 \mathrm{~b}$ (right), present a different combining site (Figure $3 b$ ). The bottom of the combining site is occupied by $\operatorname{TrpH} 103$ in Table $2 \mathrm{a}$ and by residue $\mathrm{H} 100 \mathrm{c}$ in Table $2 \mathrm{~b}$. This table can be compared to Table 2 of MacBeath and Hilvert [3], which concerns esterolytic antibodies. Residue numbering is identical to that of Kabat et al. [33], except for the three residues preceding $\mathrm{H} 101$, denoted $\mathrm{H} 100 \mathrm{a}, \mathrm{H} 100 \mathrm{~b}$ and $\mathrm{H} 100 \mathrm{c}$, according to Chothia and co-workers [34]. The CDRs consist of residues 26-32, 50-52 and 91-96 for CDRs L1, L2 and L3, respectively, and 26-32, 52-55 and 96-101 for

Waals contacts and $\pi$-stacking interactions. In addition, a few strategically placed polar functionalities, which modulate the electronic densities on key substituents in the transition state, result in enthalpic stabilisation.

\section{Other strategies}

\section{Use of a cofactor}

In order to diversify the mechanisms and introduce covalent catalysis, cofactors have been added to the antibody as an alternative to the transition-state analogue approach. The only known structure of a cofactor-dependent antibody catalyst is that of $28 \mathrm{~B} 4$, which catalyses the periodate-dependent oxidation of sulfide 28 to sulfoxide 29 (Figure 1i) [22]. This reaction was studied as a first step toward extending antibody catalysis to redox reactions. Antibody 28B4 was raised against an aminophosphonic hapten 30 that not only mimics the stereoelectronic features of the transition state, but also contains phosphonate and nitrophenyl moieties designed to provide two binding sites precisely oriented for the substrates periodate and nitroarylsulfide. The antibody stabilises developing positive
CDRs $\mathrm{H} 1, \mathrm{H} 2$ and $\mathrm{H} 3$, respectively. Common residues forming the combining site are indicated in green (residue column); residues essential for catalysis are in bold. Interactions between the antibody and hapten are coloured as follows: cation $-\pi$, brown; $\pi$-stacking, dark blue; electrostatic, gold; main van der Waals contacts, dark green; direct hydrogen bonding, red; hydrogen-bond network, violet. Residues that are probably implicated in chemical catalysis are shown in cyan. Among the few polar residues of the combining site, H35 is most often involved in the catalytic mechanism. H35 has its sidechain precisely positioned by a conserved hydrogen bond with Trp47. †The only sequence difference at the positions contacting the hapten of the germline antibody is serine at position L34. FR, framework region.

charge on sulfur and negative charge on periodate in the transition state via cation $-\pi$ and electrostatic interactions, respectively (Figure $2 \mathrm{~g}$ ). In addition, entropic restriction plays a fundamental role in catalysis through the precise alignment of the thioether substrate and oxidant.

\section{Reactive immunisation}

Catalytically active nucleophiles have rarely been selected in antibody combining sites during antibody maturation. In order to improve efficiency and widen the scope of the reactions catalysed, ways have been sought to elicit chemically reactive residues, such as nucleophiles, electrophiles and general bases or acids, directly into the antibody combining site.

Reactive immunisation is based on the use of a chemically reactive hapten designed to irreversibly modify any antibody possessing an active site group that can catalyse the reaction of interest [23]. Antibody 33F12 was obtained using $\beta$-diketone hapten 34 , which forms a Schiff base and covalently modifies the $\varepsilon$-amino group of an active site 
lysine (Figure 1j) [23]. 33F12 catalyses a vast array of aldol condensations, a $\mathrm{C}-\mathrm{C}$ bond forming reaction widely utilised in cellular metabolism and synthetic chemistry, as well as retro-aldol reactions. The broad substrate tolerance observed is the result of a lack of maturation of the immune response: because the formation of a covalent bond is the ultimate in achieving binding energy, the binding pocket does not further evolve toward higher specificity. The structure of unliganded antibody $33 \mathrm{~F} 12$ [24] has confirmed that the framework residue LysH93, which results from a somatic mutation and is essential for catalytic activity [25], is present at the base of the combining site and is probably correctly positioned to act as a nucleophile. LysH93 lies in a hydrophobic environment, so it cannot form any salt bridge or hydrogen bond with the antibody, and is uncharged. This reduces its $\mathrm{p} K_{\mathrm{a}}$ and enhances its chemical reactivity.

\section{Strategic use of haptenic charge}

Another approach that aims to generate functional residues that could participate in a specific chemical step of catalysis, also termed 'bait and switch' [26-29], uses a hapten containing a charge properly positioned to induce the required complementary charged residue. My colleagues and I recently reported the structure of the complex of antibody 4B2, which catalyses the allylic rearrangement of $\beta-\gamma$ unsaturated ketone 35 , with a short derivative of cationic hapten 37 (Figure 1k) [30]. The formation of the dienol intermediate is presumed to occur through abstraction of the $\mathrm{C} \alpha$ proton by a general base, which has been identified as GluL34 on the basis of the structural and biochemical studies. GluL34 forms a direct ionic pair interaction with the amidinium function of 36 (Figure $2 \mathrm{~h}$ ). We emphasised the advantage of the bidentate amidinium group for the precise positioning of a functional group relative to the hapten, compared with quaternary ammoniums, for which the diffuse positive charge is often stabilised by cation $-\pi$ interactions, as seen in the antibody 5C8-6 hapten complex (Figure 2b). One might think that introducing a carboxylate moiety into the hapten would, through a similar ionic interaction, induce a positively charged arginine residue in the antibody, which could act as an electrophile or a general acid in a chemical reaction. However, hapten 12 (Figure 1d), which contains two carboxylates, did not select positively charged residues in the combining site of antibody $1 \mathrm{~F} 7$, which is, moreover, not highly charged. This contrasts with chorismate mutase enzymes, which stabilise the carboxylates of chorismate by a direct ionic pair interaction with arginine residues $[13,14]$. Other structures of antibodies induced by carboxylate- or amidinium-containing haptens need to be elucidated in order to understand whether the selection of a counter-charged residue is a frequent event or not.

\section{Comparison of the combining site structures}

The top of the combining site of antibodies is formed by the CDRs. In particular, CDR loop H3 (H96-H101) is the most heavily used in antibody recognition and has a large diversity potential. Superposition of the coordinates of the catalytic antibodies that possess a small residue at position $\mathrm{H} 100 \mathrm{c}$ reveals a common cylindrical cavity, which is also present in hydrolytic antibodies [3,5,31] (Figure 3a). The absence of a bulky residue at position $\mathrm{H} 100 \mathrm{c}$ allows deep penetration of the ligands at the $\mathrm{V}_{\mathrm{L}}-\mathrm{V}_{\mathrm{H}}$ interface and explains the extensive use of framework residues in hapten binding. The common hydrophobic bottom of the cavities is probably a consequence of the similarity and hydrophobicity of the haptens used. The catalytic residues are most often polar residues of CDRs L1, L3, H1 and H3, which make up the walls of the combining site (Table 2a). Finetuning of this basic structural motif is achieved through somatic mutation and the diversity inherent to CDR H3.

When a residue with a bulky sidechain (phenylalanine or methionine) is present at position $\mathrm{H} 100 \mathrm{c}$, it fills part of the cavity and makes up the floor of the binding site (Figure 3b). The ligands bind at the upper part of the $\mathrm{V}_{\mathrm{L}}-\mathrm{V}_{\mathrm{H}}$ interface, leading to a pocket in which the bottom is formed by residues of the CDRs, instead of the framework. This may lead to a combining site that is much shallower than that in Figure $3 \mathrm{a}$, as found in $7 \mathrm{G} 12$ (Figure $3 \mathrm{~b}$ ) and 1F7. The positions of the haptens in the combining sites are less similar and the shapes of the cavities are more diversified because the fewer common residues that make up the combining site principally belong to the hypervariable loops (Table 2b).

The importance of residue $\mathrm{H} 100 \mathrm{c}$ in determining the shape of the binding cavity suggests that its mutagenesis may confer on the antibodies new catalytic properties.

\section{Conclusions}

A comparison of the structures of nonhydrolytic antibody catalysts published during the period 1998 to the present has shown that these antibodies use catalytic pockets of different shapes, depending on the nature of residue H100c. Antibodies with a small or no residue at position H100c present a structurally similar, deep combining site, with hydrophobic residues of the framework making up the floor. When a bulky residue occupies position $\mathrm{H} 100$ c, it takes up part of the bottom of the cavity; the binding site is formed almost exclusively by the CDRs and adopts various shapes.

The apolar bottom of the combining site of catalytic antibodies serves to exclude the solvent and acts as a template to bind the substrate(s) in a reactive conformation, whereas the more polar residues that form the walls of the cavity play a catalytic role through hydrogen-bonding, cation $-\pi$ or electrostatic interactions. These pockets are appropriate to govern the regiochemistry and stereochemistry of the reaction, as demonstrated by the control of the fate of intermediates. Moreover, the antibodies often demonstrate stereoselectivity, although no stereochemical information was programmed into the hapten. Hence, antibody catalysts will remain particularly useful to catalyse disfavoured reactions. 
Immunological selection based on transition-state analogues results in noncovalent catalysis and narrow substrate specificity. On the other hand, other strategies, such as 'bait and switch' or reactive immunisation, are more appropriate for reactions that necessitate a reactive residue such as a nucleophile or a base/acid. In these new strategies, the hapten does not need to match precisely the transition state of the reaction, but rather generates a functional, strategically positioned residue. This confers on these methods the advantage of generating antibodies with broad substrate tolerance that even catalyse different reactions proceeding through the same mechanism (Table 1). This is especially useful when transition-state analogues are difficult to synthesise, when the structures of the substrate and the transition state are not very different, or when the mechanism of the reaction involves multiple intermediates or transition states.

Improvements in hapten design and screening of antibodies, together with structure-based mutagenesis experiments and random mutagenesis coupled with genetic selection, will, in the future, lead to more efficient antibodies that combine transition-state stabilisation and chemical reactivity.

\section{Update}

Antibody 21D8 raised against a 1,5-naphtalene disulfonate hapten catalyses the solvent-sensitive decarboxylation of 3-carboxybenzisoxasoles $\left(\mathrm{k}_{\mathrm{cat}} / \mathrm{K}_{\mathrm{m}}=1.1 \times 104 \mathrm{M}^{-1} \mathrm{~min}^{-1}\right.$; $\left.\mathrm{k}_{\mathrm{cat}}=57.6 \mathrm{~min}^{-1} ; \mathrm{k}_{\mathrm{cat}} / \mathrm{k}_{\mathrm{non}}=61,000\right)$. The antibody-hapten complex structure has recently been determined at $1.61 \AA$ resolution $(\mathrm{PDB}$ code $1 \mathrm{c} 5 \mathrm{c}$ ) [35]. Antibody 21D8 possesses an unusually short $\mathrm{H} 3$ loop with no residue at

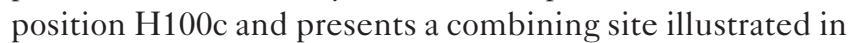
Figure $3 \mathrm{a}$, with two anionic binding sites for the two sulfonate groups of the hapten. The sidechains of ArgL96 and HisH35 constitute one site, whereas the other is constructed from the sidechains of ArgL46, TrpH103, SerL36 and the backbone amide group of AlaH101. Although the authors computationally docked the substrate and the transition state into 21D8, it was not possible to identify unambiguously in which of the sulfonate-binding sites the carboxylate of the substrate binds. However, one docking mode shows a specific hydrogen bond between the oxygen of the breaking N-O bond and ArgL46 that could stabilise the transition state. Binding throughout the decarboxylation reaction is therefore realised through electrostatic and hydrogen-bonding interactions and by burying the aromatic portion of the substrate in the hydrophobic slot at the bottom of the combining site. Whereas water stabilises the localised charge of the substrate much more than the delocalised charge of the transition state, the antibody is presumed to accelerate the reaction by stabilising the substrate and the transition state to a similar extent, as in aprotic solvents.

\section{Acknowledgements}

I thank all my colleagues who read the manuscript: B Badet, B Gigant, J Janin, M Knossow, J-P Mahy and C Tellier.

\section{References}

1. Schultz PG, Lerner RA: From molecular diversity to catalysis: lessons from the immune system. Science 1995, 269:1835-1842.

2. Blackburn GM, Datta A, Denham H, Wentworth P: Catalytic antibodies. Adv Phys Org Chem 1998, 31:249-392.

3. MacBeath $G$, Hilvert D: Hydrolytic antibodies: variation on a theme Chem Biol 1996, 3:433-445.

4. Wade $\mathrm{H}, \mathrm{Scanlan}$ TS: The structural and functional basis of antibody catalysis. Annu Rev Biophys Biomol Struct 1997, 26:461-493.

5. Charbonnier J-B, Gigant B, Golinelli-Pimpaneau B, Knossow M: Similarities of hydrolytic antibodies revealed by their X-ray structures: a review. Biochimie 1997, 79:653-660.

6. Hilvert D, MacBeath G, Shin JA: The structural basis of antibody catalysis. In Bioorganic Chemistry: Peptides and Proteins. Edited by Hecht SM. New York: Oxford University Press; 1998:355-366.

7. Romesberg FE, Santarsiero BD, Spiller B, Yin J, Barnes D, Schultz PG, Stevens RC: Structural and kinetic evidence for strain in biological catalysis. Biochemistry 1998, 37:14404-14409.

8. Gruber K, Zhou B, Houk KN, Lerner RA, Shevlin CG, Wilson IA: Structural basis for antibody catalysis of a disfavored ring closure reaction. Biochemistry 1999, 38:7062-7074.

9. Li T, Lerner RA, Janda KD: Antibody-catalyzed cationic reactions: rerouting of chemical transformations via antibody catalysis. Acc Chem Res 1997, 30:115-121.

10. Paschall CM, Hasserodt J, Jones T, Lerner RA, Janda KD, Christianson DW: Convergence of catalytic antibody and terpene cyclase mechanisms : polyene cyclisation directed by carbocation- $\pi$ interactions. Angew Chem Int Ed Engl 1999, 38:1743-1747.

11. Laschat S: Pericyclic reactions in biological systems. Does Nature know about the Diels-Alder reaction? Angew Chem Int Ed Engl 1996, 35:289-291.

12. Haynes MR, Stura EA, Hilvert D, Wilson IA: Routes to catalysis: structure of a catalytic antibody and comparison with its natural counterpart. Science 1994, 263:646-652.

13. Chook YM, Hengming K, Lipscomb WN: Crystal structures of the monofunctional chorismate mutase from Bacillus subtilis and its complex with a transition state analog. Proc Natl Acad Sci USA 1993, 90:8600-8603.

14. Lee AY, Karplus PA, Ganem B, Clardy J: Atomic structure of the buried catalytic pocket of Escherichia coli chorismate mutase. J Am Chem Soc 1995, 117:3627-3628.

15. Ulrich HD, Mundorff E, Santarsiero BD, Driggers EM, Stevens RC, Schultz PG: The interplay between binding energy and catalysis in the evolution of a catalytic antibody. Nature 1997 , 389:271-274.

16. Mundorff EC, Hansin MA, Varvak A, Ulrich H, Schultz PG, Stevens RC: Conformational effects in biological catalysis: an antibody-catalyzed oxy-Cope rearrangement. Biochemistry 2000, 39:627-632.

17. Romesberg FE, Spiller B, Schultz PG, Stevens RC: Immunological origins of binding and catalysis in a Diels-alderase antibody. Science 1998, 279:1929-1933.

18. Heine A, Stura EA, Yli-Kauhaluoma JT, Gao C, Deng Q, Beno BR, Houk KN, Janda KD, Wilson IA: An antibody exo Diels-alderase inhibitor complex at 1.95 Angstrom resolution. Science 1998, 279:1934-1940.

19. Xu J, Deng $Q$, Chen J, Houk KN, Bartek J, Hilvert D, Wilson IA: Evolution of shape complementarity and catalytic efficiency from a primordial antibody template. Science 1999 , 286:2345-2348.

20. Campbell AP, Tarasow TM, Massefski W, Wright PE, Hilvert D: Binding of a high-energy substrate conformer in antibody catalysis. Proc Natl Acad Sci USA 1993, 99:8663-8667.

21. Driggers EM, Cho HS, Liu CW, Katzka CP, Braisted AC, Ulrich HD, Wemmer DE, Schultz PG: Mechanistic studies of an antibodycatalyzed pericyclic rearrangement. J Am Chem Soc 1998 , 120:1945-1958. 
22. Hsieh-Wilson LC, Schultz PG, Stevens RC: Insights into antibody catalysis: structure of an oxygenation catalyst at $1.9 \AA$ resolution. Proc Natl Acad Sci USA 1996, 93:5363-5367.

23. Wagner J, Lerner RA, Barbas CF III: Efficient aldolase catalytic antibodies that use the enamine mechanism of natural enzymes. Science 1995, 270:1797-1800.

24. Barbas CF III, Heine A, Zhong G, Hoffman T, Gramatikova S, Björnestedt R, List B, Anderson J, Stura EA, Wilson IA, Lerner RA: Immune versus natural selection: antibody aldolases with enzymic rates but broader scope. Science 1997, 278:2085-2092.

25. Karlstrom A, Zhong G, Rader C, Larsen NA, Heine A, Fuller R, List B, Tanaka F, Wilson IA, Barbas CF III, Lerner RA: Using antibody catalysis to study the outcome of multiple evolutionary trials of a chemical task. Proc Natl Acad Sci USA 2000, 97:3878-3883.

26. Shokat KM, Leumann CJ, Sugasawara R, Schultz PG: A new strategy for the generation of catalytic antibodies. Nature 1989, 338:269-271.

27. Janda KD, Weinhouse MI, Schloeder DM, Lerner RA, Benkovic SJ: Bait and switch strategy for obtaining catalytic antibodies with acyl-transfer capabilities. J Am Chem Soc 1990, 112:1274-1275.

28. Janda KD, Weinhouse MI, Danon T, Pacelli KA, Schloeder DM: Antibody bait and switch catalysis: a survey of antigens capable of inducing abzymes with acyl-transfer properties. J Am Chem Soc 1991, 113:5427-5434.
29. Thorn SN, Daniels RG, Auditor MT, Hilvert D: Large accelerations in antibody catalysis by strategic use of haptenic charge. Nature 1995, 373:228-230.

30. Golinelli-Pimpaneau B, Gonçalves O, Dintinger T, Blanchard D, Knossow M, Tellier C: Structural evidence for a programmed general base in the active site of a catalytic antibody. Proc Natl Acad Sci USA 2000, 97:9892-9895.

31. Charbonnier J-B, Carpenter E, Gigant B, Golinelli-Pimpaneau B Tawfik D, Eshhar Z, Green BS, Knossow M: Crystal structure of the complex of a catalytic antibody Fab fragment with a transition state analog: structural similarities in esterase-like catalytic antibodies. Proc Natl Acad Sci USA 1995, 92:11721-11725.

32. Gigant B, Tsumuraya T, Fujii I, Knossow M: Diverse structural solutions to catalysis in a family of antibodies. Structure 1999 , 7:1385-1393.

33. Kabat EA, Wu TT, Perry HM, Gottesman KS, Foeller C: Sequences of Proteins of Immunological Interest. Bethesda, MD: US Public Health Service, National Institutes of Health; 1991.

34. Morea V, Tramontano A, Rustici M, Chothia C, Lesk AM: Conformations of the third hypervariable region in the $\mathrm{V}_{\mathrm{H}}$ domain of immunoglobulins. J Mol Biol 1998, 257:269-294.

35. Hotta K, Lange H, Tantillo DJ, Houk KN, Hilvert D, Wilson IA : Catalysis of decarboxylation by a preorganized heterogeneous microenvironment: crystal structures of abzyme 21D8. J Mol Biol 2000, 302:1213-1225. 